\title{
Tagged spectator DIS on a polarized spin-1 target
}

\section{W. Cosyn ${ }^{* a}$ M. Sargsian, ${ }^{b}$ C. Weiss ${ }^{c}$}

a Dept. of Physics and Astronomy, Ghent University, Proeftuinstraat 86, B9000 Ghent, Belgium

b Department of Physics, Florida International University, Miami, FL 33199, USA

c Theory Center, Jefferson Lab, Newport News, VA 23606, USA

E-mail: wim.cosyn@ugent.be, sargsian@fiu.edu, weiss@jlab.org

\begin{abstract}
We discuss the process of deep-inelastic electron scattering (DIS) on the polarized deuteron with detection of a nucleon in the nuclear fragmentation region ("spectator tagging"). We cover (a) the general structure of the DIS cross section; (b) the structure functions of the process in the impulse approximation using deuteron light-front wave functions; (c) the extraction of free neutron structure by extracting the residue of observables at the free nucleon pole ("pole extrapolation"). As an application we consider the extraction of the free neutron structure function $g_{1 n}$ through polarized electron scattering on the longitudinally polarized deuteron with proton tagging and on-shell extrapolation in the recoil momentum, possible at an EIC with polarized deuteron beams and forward detectors.
\end{abstract}

XXIV International Workshop on Deep-Inelastic Scattering and Related Subjects

11-15 April, 2016

DESY Hamburg, Germany

\footnotetext{
* Speaker.
} 


\section{Introduction}

We consider the process of tagged spectator DIS process on a deuteron target:

$$
e(l)+D\left(P_{D}\right) \rightarrow e^{\prime}\left(l^{\prime}\right)+X\left(P_{X}\right)+N\left(p_{N}\right)
$$

where a recoil nucleon is detected in the final state with low momentum, $\sim$ few $100 \mathrm{MeV}$ in the deuteron rest frame. Compared to more conventional inclusive deuteron DIS measurements, where only the scattered electron is measured, this reaction has the advantage that one has more control over the initial nuclear configuration of the deuteron target and the active nucleon (proton, neutron) is positively identified, thereby eliminating dilution from scattering on the other nucleon. In inclusive measurements the struck nucleon has Fermi motion and one has to average over all nuclear configurations, as well as account for possible non-nucleonic components in the wave function. Especially at high Bjorken $x$, the Fermi motion affects the uncertainties in the extraction of neutron structure functions and flavor separation of parton distribution functions. Non-nucleonic components are suppressed in reaction (1.1) because of the deuteron target (isospin forbids $N \Delta$ components) and nucleon tagging ( $\Delta \Delta$ contribution is eliminated).

The tagged spectator deuteron process has been measured in two pioneering experiments at Jefferson Lab (JLab). The Deeps experiment [1] measured at proton momenta $300-600 \mathrm{MeV}$, while the BONuS experiment $[2,3]$ measured down to momenta of $70 \mathrm{MeV}$. Model calculations including the effect of final-state interactions (FSI) are available [4, 5]. A follow-up experiment is planned at the $12 \mathrm{GeV}$ JLab upgrade [6] and another experiment will use the process to study the recoil momentum and isospin dependence of the EMC effect [7] as the tagged nucleon offers control over the off-shellness of the struck nucleon and the density of the nucleus configuration.

Spectator tagging also enables the application of the so-called pole extrapolation [8], where the cross section is extrapolated to the pole $\left[t=\left(p_{D}-p_{N}\right)^{2}=p_{i}^{2}=m_{N}^{2}\right]$ of the propagator of the $t$-channel nucleon (see Fig. 1a), and one can effectively probe on-shell neutron structure when tagging a proton and extracting the residue of the cross section at the pole. Due to the small binding energy of the deuteron the actual extrapolation length into unphysical kinematics is very small and corresponds to almost zero but imaginary spectator momentum $p_{N}$ in the deuteron rest frame. Pole extrapolation also has the advantage that the FSI contribution cancels as it does not have a pole for the on-shell exchange due to the loop momentum integration [8]. This makes pole extrapolation a very powerful, model-independent method of extracting neutron structure. Recently, pole extrapolation was applied to the BONuS data and a surprising rise in the $F_{2 n} / F_{2 p}$ ratio at high $x$ was observed, albeit at sub-DIS values of $Q^{2}$ [9].

Deuteron DIS with spectator tagging will be possible at a future EIC. In such measurements, the spectator nucleon moves forward with $\sim 1 / 2$ of the beam momentum and is detected with forward detectors. Moreover, in a collider there is no target material. Compared to a fixed target setup, where the detectors have to be very close to the target and physically small (which limits their performance), this makes detection of the spectators more straightforward. In particular, an EIC could enable tagged DIS with polarized deuteron beams. These beams would be available in the Jefferson Lab EIC (JLEIC) figure-8 ring design $[10,11]$ and allow for the measurement of neutron spin structure over a wide kinematic domain. The small extrapolation length for the tagged spectator 
process also means the $D$-wave admixture is very small and deuteron polarization is almost $100 \%$ transferred to the nucleons.

In these proceedings, we summarize the theoretical formalism for tagging on a polarized deuteron and show estimates for the vector polarized double spin asymmetry in kinematics accessible at an EIC. The full formalism will be presented in Ref. [12] and the work presented here is part of a dedicated LDRD program at JLAB providing simulations for an EIC [13].

\section{Formalism}

To compute observables of reaction (1.1) with polarized initial beams, the general form of the SIDIS spin-1 cross section can be used [12], as detecting a recoil nucleon can be considered SIDIS in the target fragmentation region. The spin- 1 target ensemble is described by a 3 by 3 density matrix $\rho_{\lambda \lambda^{\prime}}$, which has 8 parameters (3 vector, 5 tensor polarization) [14]. The hadronic tensor is written as

$$
W^{\mu v}=\sum_{\lambda^{\prime} \lambda} \rho_{\lambda \lambda^{\prime} W^{\mu v}}\left(\lambda^{\prime}, \lambda\right)
$$

and obeys the transversality conditions $q_{\mu} W^{\mu v}=W^{\mu v} q_{v}=0$ and parity invariance constraints. The cross section has 41 structure functions each with an azimuthal modulation depending on the angle of the recoil nucleon and parameters of the density matrix [12]:

$$
\frac{d \sigma}{d x d Q^{2} d \phi_{l^{\prime}}}=\frac{y^{2} \alpha_{\mathrm{em}}^{2}}{Q^{4}(1-\varepsilon)}\left(\mathscr{F}_{U}+\mathscr{F}_{S}+\mathscr{F}_{T}\right) d \Gamma_{P_{N}}
$$

The 5 upolarized (contained in $\mathscr{F}_{U}$ ) and 13 vector polarized (present in $\mathscr{F}_{S}$ ) terms have identical azimuthal and vector polarization dependence as the spin 1/2 case [15], while the 23 structure functions contained in $\mathscr{F}_{T}$ are unique to the spin 1 case and will be presented in detail elsewhere [12].

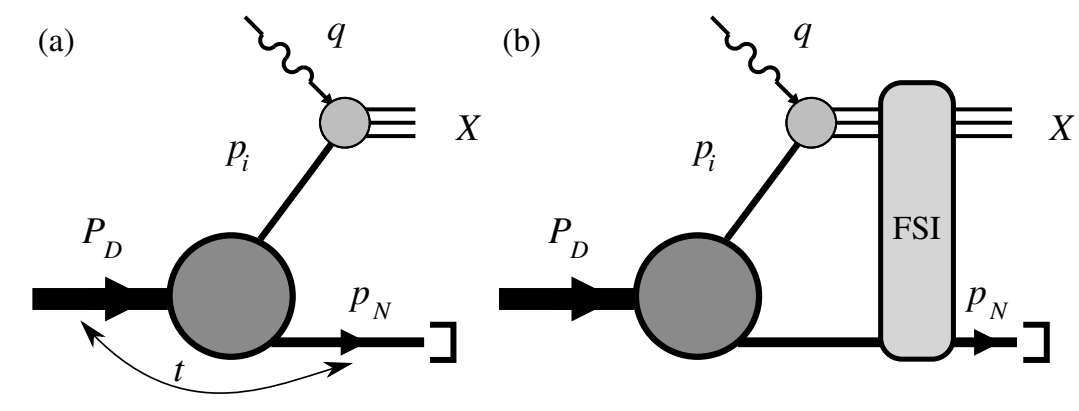

Figure 1: Impulse approximation (a) and FSI (b) of the tagged spectator DIS process on a composite deuteron target.

A dynamical model for reaction (1.1), suitable for high-energy probes, is used where the nuclear structure is factorized from the probe-nucleon interaction [12]. We show results in the impulse approximation (IA) depicted in Fig. 1a and do not include the FSI contribution of Fig. 1b as it has different analytic properties compared to the IA and does not contribute at the on-shell nucleon pole. In the IA, the hadronic tensor reads [12]

$$
W_{D}^{\mu v}\left(\lambda^{\prime}, \lambda\right)=4(2 \pi)^{3} \frac{\alpha_{N}}{2-\alpha_{N}} \sum_{i=U, z, x, y} W_{N, i}^{\mu v} \rho_{D}^{i}\left(\lambda^{\prime}, \lambda\right)
$$


where $\alpha_{N}=2 p_{N}^{+} / P_{D}^{+}$is the recoil nucleon lightcone momentum fraction, the $W_{N, i}^{\mu v}$ depend on structure functions of the struck nucleon $F_{1 N}, F_{2 N}, g_{1 N}, g_{2 N}$ evaluated at $\tilde{x} \approx x /\left(2-\alpha_{N}\right)$ and $Q^{2}$, and $\rho_{D}^{i}\left(\lambda^{\prime}, \lambda\right)$ are deuteron light-front densities depending on the deuteron light-front wave function. In high-energy scattering it is natural to describe deuteron structure using the light-front wave function, as in this formulation the off-shellness of the $N N$ component remains finite in the highenergy limit, which is not the case in the instant form. The light-front deuteron wave function $\Psi_{\lambda}^{D}\left(\boldsymbol{k}_{f}, \lambda_{1}, \lambda_{2}\right)$ has the same structure and rotational invariance properties as the non-relativistic wave function [16, 17, 18], but differs in two important aspects: a) there are Melosh rotations $R_{f c}$ accounting for relativistic spin; b) it depends on a dynamical three-momentum $\boldsymbol{k}_{f}$ which is related to the kinematical momenta of the target deuteron and recoil nucleon $p_{N}$ in the following manner:

$$
\frac{k_{f}^{z}}{E_{k_{f}}}=1-\alpha_{N}, \quad \boldsymbol{k}_{f}^{\perp}=-\boldsymbol{p}_{N}^{\perp}+\frac{\alpha_{i}}{2} \boldsymbol{P}_{D}^{\perp}, \quad E_{k}=\frac{m_{N}^{2}+\boldsymbol{k}_{f}^{\perp 2}}{\alpha_{N}\left(2-\alpha_{N}\right)} .
$$

The light-front wave function reads

$$
\Psi_{\lambda}^{D}\left(\boldsymbol{k}_{f}, \lambda_{1}, \lambda_{2}\right)=\sqrt{E_{k_{f}}} \sum_{\lambda_{1}^{\prime} \lambda_{2}^{\prime}} \mathscr{D}_{\lambda_{1} \lambda_{1}^{\prime}}^{\frac{1}{2}}\left[R_{f c}\left(k_{1_{f}}^{\mu} / m_{N}\right)\right] \mathscr{D}_{\lambda_{2} \lambda_{2}^{\prime}}^{\frac{1}{2}}\left[R_{f c}\left(k_{2_{f}}^{\mu} / m_{N}\right)\right] \Phi_{\lambda}^{D}\left(\boldsymbol{k}_{f}, \lambda_{1}^{\prime}, \lambda_{2}^{\prime}\right),
$$

where $\Phi_{\lambda}^{D}\left(\boldsymbol{k}_{f}, \lambda_{1}^{\prime}, \lambda_{2}^{\prime}\right)$ has the structure of a non-relativistic deuteron wave function, and $\mathscr{D}_{\lambda \lambda^{\prime}}^{\frac{1}{2}}$ denote the Wigner D-matrices.

Using Eq. (2.3) it is possible to write all 41 structure functions that appear in Eq. (2.2) as a linear combination of the tensor and polarization components of $W_{D}^{\mu v}\left(\lambda^{\prime}, \lambda\right)[12]$.

\section{Results}

As an observable of interest in the tagged spectator process, we consider the double spin asymmetry for a longitudinally vector polarized target:

$$
A_{\|}=\frac{\sigma(++)-\sigma(+-)-\sigma(-+)+\sigma(--)}{\sigma(++)+\sigma(+-)+\sigma(-+)+\sigma(--)}=\frac{F_{L S_{L}}}{F_{U U, T}+\varepsilon F_{U U, L}}
$$

where $\sigma\left(\lambda_{e}, \lambda_{D}\right)$ denotes the cross section for spin projections $\lambda_{e}= \pm \frac{1}{2}, \lambda_{D}= \pm 1$, averaged over the azimuthal angle of the recoil proton. The $F_{i}$ are structure functions appearing in the cross section [12,15], for which closed expressions have been obtained in the IA model [12]. We can schematically write the structure function appearing in the nominator of $A_{\|}$as a product of four components:

$$
\begin{aligned}
F_{L S_{L}}=\{ & \text { kin. factors and deuteron polarization }\} \times\left\{g_{1}\left(\tilde{x}, Q^{2}\right), g_{2}\left(\tilde{x}, Q^{2}\right)\right\} \\
& \times\{\text { bilinear forms in deuteron radial wave function } U(k), W(k)\} \\
& \times\{\text { rel. spin factors resulting from Melosh transf. depending on } \boldsymbol{k}\},
\end{aligned}
$$

where $U(k)[W(k)]$ is the deuteron radial $S$-wave [D-wave]. The $F_{U U, T}$ and $F_{U U, L}$ functions in the denominator have a similar form but are spin-independent so involve the nucleon structure functions $F_{1 N}, F_{2 N}$ in the second component and omit the last component in Eq. (3.2) (Details will be given in Ref. [12]). 


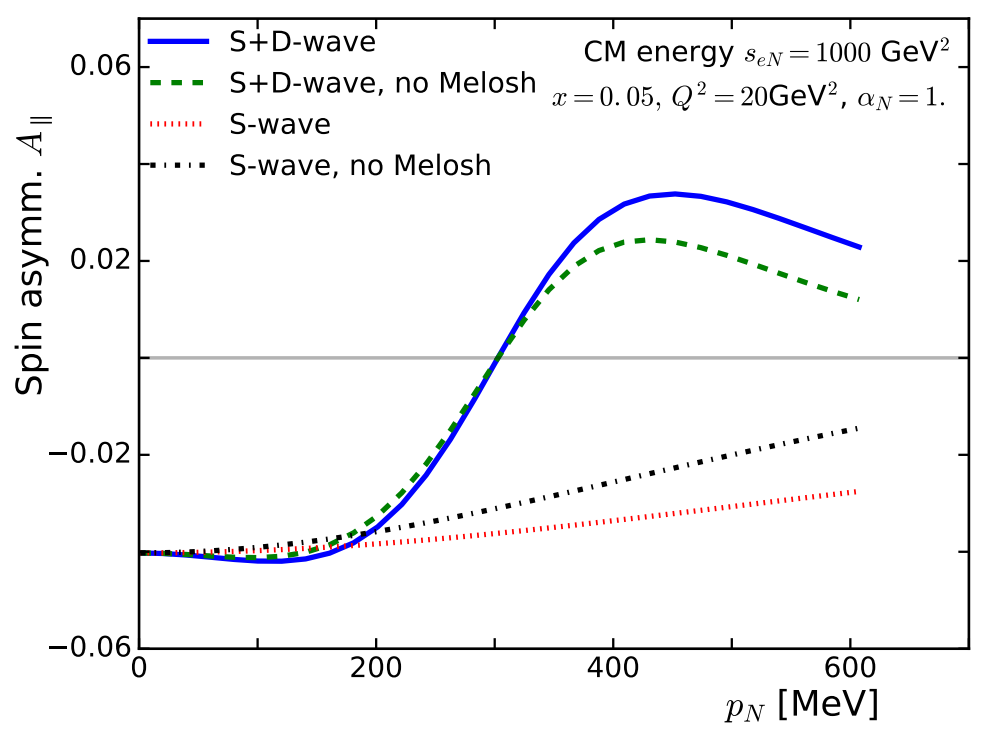

Figure 2: Spectator momentum dependence of the double spin asymmetry $A_{\|}$as a function of the spectator proton momentum in the deuteron rest frame. Full blue curve includes both the $S$ - and $D$-wave deuteron components and accounts for the Melosh rotations in the light-front wave function, dashed green curve only includes the deuteron $S$-wave component and accounts for the Melosh rotations in the light-front wave function. The black dashed-dotted curve (only $S$-wave component) and red dotted curve ( $S$ - and $D$-wave components) do not include the Melosh rotations in the wave function.

Pole extrapolation for $A_{\|}$is straightforward as the nucleon pole $1 /\left(m_{N}^{2}-t\right)^{2}$ in the cross sections, which appears in $A_{\|}$through the radial deuteron wave function dependence $[U(k), W(k)$ ] of all structure functions [Eq. (3.2)], cancels between the numerator and denominator. Experimentally, the ratio has the advantage that systematic errors largely cancel. When performing pole extrapolation on $A_{\|}$, one obtains the result

$$
A_{\|} \quad \text { on-shell } \sim D \frac{g_{1 n}}{F_{1 n}},
$$

where $D$ is the depolarization factor, which emerges from the kinematic factors in the cross section. Then the on-shell $A_{\|}$together with data for $F_{1 n}$ allows one to extract on-shell neutron spin structure. Figure 2 shows a calculation of $A_{\|}$as a function of recoil proton momentum at kinematics accessible at an EIC and illustrates the influence of the deuteron D-wave and the Melosh rotations in Eq. (3.2). One clearly observes the influence of the $D$-wave (with largest size effects around the tensor force dominated region of 300-500 MeV) and also the influence of the Melosh rotations for higher recoil momenta. Both effects effectively drop out towards the on-shell nucleon pole and the asymmetry is quite flat at recoil momenta $\leq 100 \mathrm{MeV}$, making the extrapolation for the IA robust.

\section{Summary}

In sum, spectator tagging with polarized deuteron at EIC would enable precision measurements of neutron spin structure over a wide kinematic range. Further R\&D are needed to extend the theoretical framework (final-state interactions, nuclear shadowing effects) and realize the full potential of this method. 


\section{References}

[1] CLAS collaboration, A. V. Klimenko et al., Electron scattering from high-momentum neutrons in deuterium, Phys. Rev. C73 (2006) 035212, [nucl-ex/ 0510032 ].

[2] CLAS collaboration, N. Baillie, S. Tkachenko, J. Zhang, P. Bosted, S. Bültmann, M. E. Christy et al., Measurement of the neutron F2 structure function via spectator tagging with CLAS, Phys.Rev.Lett. 108 (2012) 199902, [1110.2770].

[3] CLAS collaboration, S. Tkachenko et al., Measurement of the structure function of the nearly free neutron using spectator tagging in inelastic ${ }^{2} H(e, e$ 'p)X scattering with CLAS, Phys. Rev. C89 (2014) 045206, [1 402 . 2477].

[4] W. Cosyn and M. Sargsian, Final-state interactions in semi-inclusive deep inelastic scattering off the Deuteron, Phys. Rev. C84 (2011) 014601, [1012.0293].

[5] V. Palli, C. Ciofi degli Atti, L. P. Kaptari, C. B. Mezzetti and M. Alvioli, Slow Proton Production in Semi-Inclusive Deep Inelastic Scattering off Deuteron and Complex Nuclei: Hadronization and Final State Interaction Effects, Phys. Rev. C80 (2009) 054610, [0 911.1377$].$

[6] S. Bueltmann et al., The Structure of the Free Neutron at Large x-Bjorken [JLAB-PR12-06-113].

[7] O. Hen, L. B. Weinstein, S. Gilad and S. A. Wood, In Medium Nucleon Structure Functions, SRC, and the EMC effect, 1409.1717.

[8] M. Sargsian and M. Strikman, Model independent method for determination of the DIS structure of free neutron, Phys. Lett. B639 (2006) 223-231, [hep-ph/ 0511054 ].

[9] W. Cosyn and M. M. Sargsian, High x Structure Function of the Virtually Free Neutron, Phys. Rev. C93 (2016) 055205, [1506.01067].

[10] W. Cosyn, V. Guzey, D. W. Higinbotham, C. Hyde, S. Kuhn, P. Nadel-Turonski et al., Neutron spin structure with polarized deuterons and spectator proton tagging at EIC, J. Phys. Conf. Ser. 543 (2014) 012007, [1409.5768].

[11] W. Cosyn, V. Guzey, M. Sargsian, M. Strikman and C. Weiss, Electron-deuteron DIS with spectator tagging at EIC: Development of theoretical framework, EPJ Web Conf. 112 (2016) 01022, [1601.06665].

[12] W. Cosyn, M. Sargsian, C. Weiss, in preparation.

[13] C. Weiss et al., Physics potential of polarized light ions with EIC@JLab, https://eic.jlab.org/wiki/index.php/Forward_Tagging.

[14] E. Leader, Spin in Particle Physics (Cambridge Monographs on Particle Physics, Nuclear Physics and Cosmology). Cambridge University Press, Oct., 2005.

[15] A. Bacchetta, M. Diehl, K. Goeke, A. Metz, P. J. Mulders and M. Schlegel, Semi-inclusive deep inelastic scattering at small transverse momentum, JHEP 02 (2007) 093, [hep-ph/ 0611265 ].

[16] L. L. Frankfurt and M. I. Strikman, High-Energy Phenomena, Short Range Nuclear Structure and QCD, Phys. Rept. 76 (1981) 215-347.

[17] B. Keister and W. Polyzou, Relativistic Hamiltonian dynamics in nuclear and particle physics, Adv.Nucl.Phys. 20 (1991) 225-479.

[18] P. L. Chung, W. N. Polyzou, F. Coester and B. D. Keister, Hamiltonian Light Front Dynamics of Elastic electron Deuteron Scattering, Phys. Rev. C37 (1988) 2000-2015. 